\title{
Norepinephrine transporter (NET) as a predictive marker of response to PI3K/mTOR inhibition in pheochromocytoma
}

Ninelia Minaskan, Misu Lee, Tobias Wiedemann, Martin Irmler, Johannes Beckers, Rickmer Braren, Behrooz H. Yousefi, Iina Laitinen, Natalia S. Pellegata

Institute for Diabetes and Cancer, Institute of Experimental Genetics, Helmholtz Zentrum München, Neuherberg, Germany; Institute of Radiology, Klinikum rechts der Isar, Technische Universität München, Munich, Germany; Department of Pharmaceutical Radiochemistry, Technische Universität München, Garching, Germany; Department of Nuclear Medicine, Technische Universität

\section{Abstract:}

Pheochromocytomas (PCs) are rare highly vascularized neuroendocrine tumors derived from neural crest-derived chromaffin cells located ether in adrenal medulla or sympathetic ganglia (paragangliomas). Although most cases are benign but about $10 \%$ of all PCs can become malignant and resistant to conventional chemotherapy or radiotherapy. Therefore, novel therapeutic approaches are required. In this study, we verified the antitumor efficacy of the dual PI3K/mTOR inhibitor BEZ235 in a unique model of bilateral PCs, MENX affected rats (Figure 1) and identified molecular read outs of drus treatment Genome-wide transcriptome profiling of PCs from drug-treated or placebo-treated rats was conducted, and identified the S/c6a2 gene, encoding the NET protein, as a target of BEZ235, which is inhibited by drug treatment in a dose-dependent manner. Functional analyses confirmed a predictive role for NET expression in the response to PI3K/mTOR inhibition, which can be monitored using NETselective functional positron emission tomography (PET) imaging with 18F-LMI1195. Moreover, BEZ235 reduced Slc6a2/NET expression also in PC cell line, MPC.

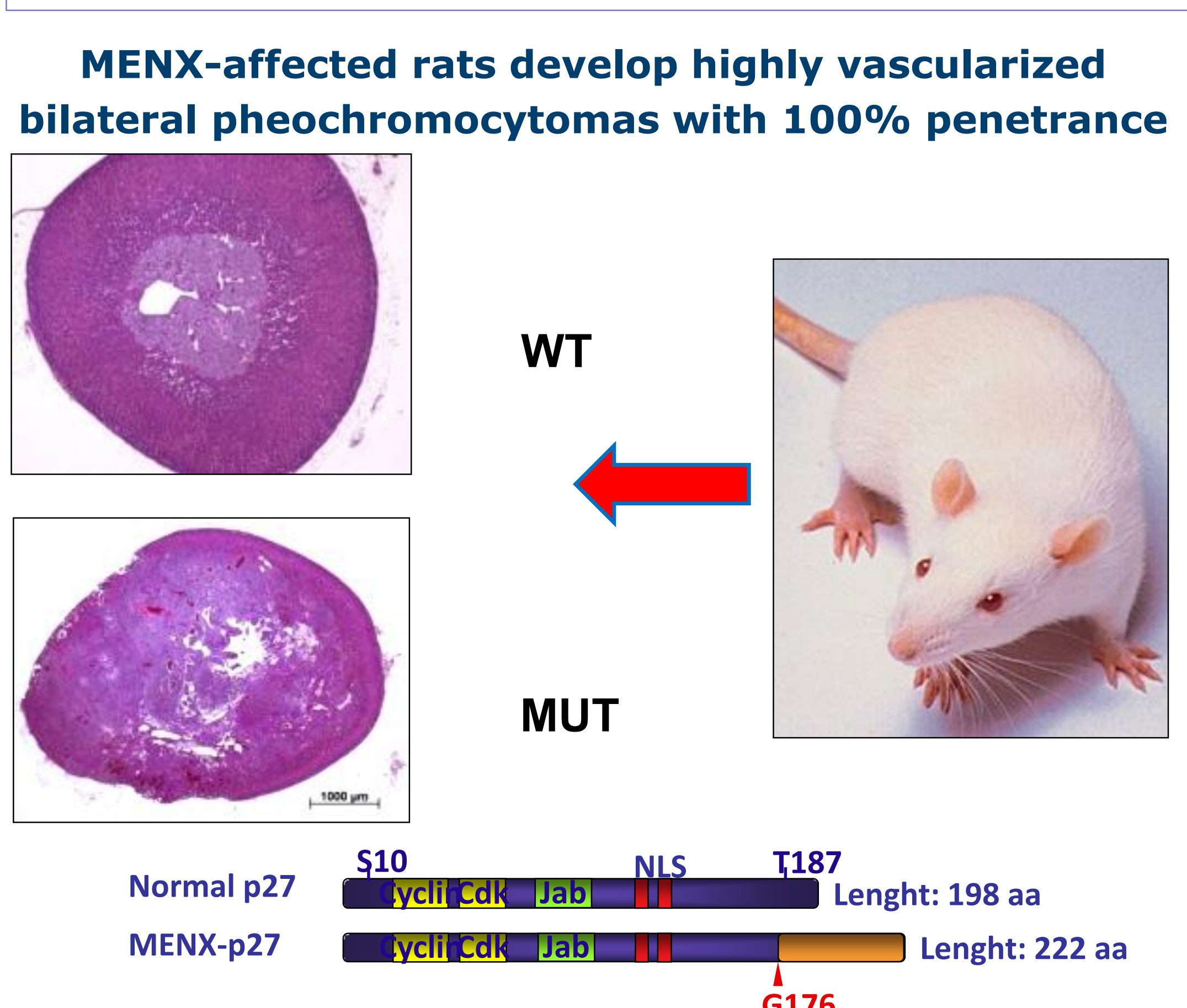

Figure 1. MENX-ra

\section{Material and Methods}

BEZ235:

Rat PC tissue samples:

Sprague-Dawley rats

mmunostainin:

Primary antibodies directed against phospho S6 (S6-S240/244; Cell Signaling Technology), p-AKT (Ser473; Cell Signaling Technology), Ki67 (Dako), activated caspase-3 (Cell Signaling Technology) (PET)

$\mathrm{NT}^{2}$ profiler PCR array, semiquantitative RT-PCR and quantitative TaqMan RT-PCR:

Cell proliferation and apoptosis assays:

Cell proliferation was measured with WS

Magnetic resonance imagin:

MRI was performed using a 3.0 Tesla clinical MRI system. T2-weighted (T2w) turbo spin echo sequence was performed to assess the tumor volume before and after treatment. Diffusion weighted-MRI (DW-MRI) was perfor

\section{Results}

PI3K/mTOR inhibition shows dose-dependent effects on cell proliferation, cell death and angiogenesis in a model of endogenous PCCs in vivo.

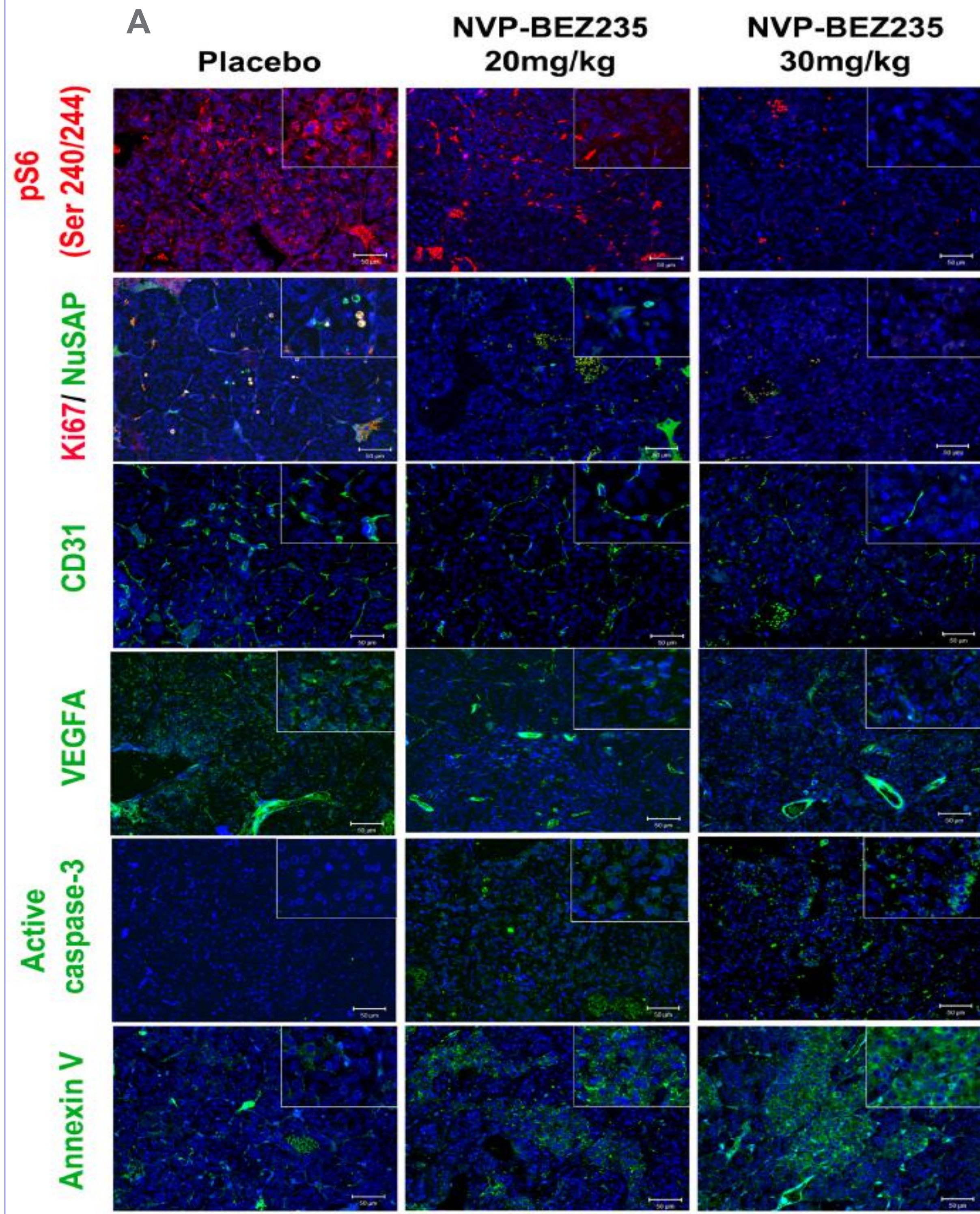

NET expression is downregulated in MPC cells after BEZ235 treatment

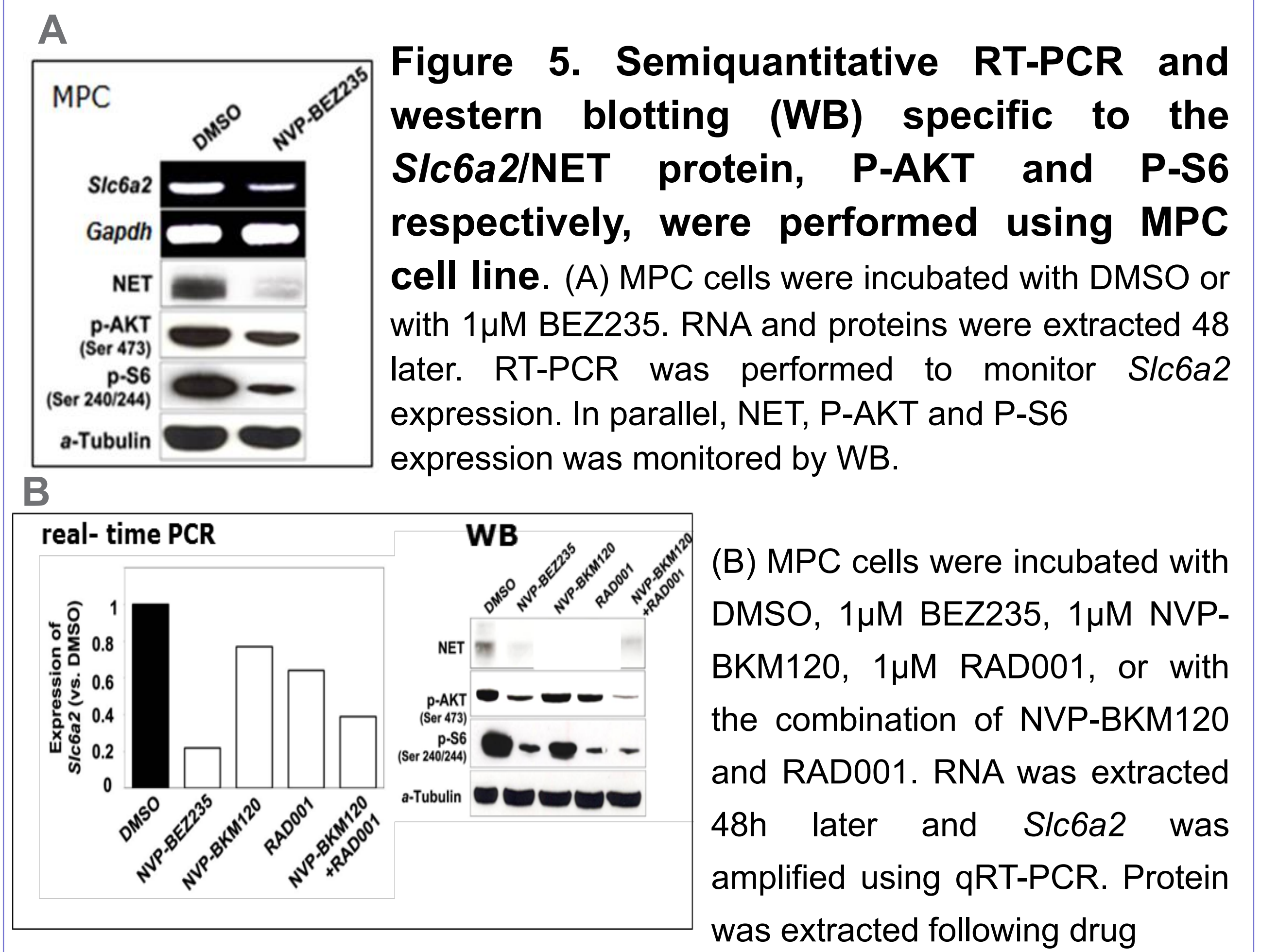

Figure 2. Analysis of PCC tissues from rats treated with placebo or BEZ235.

(A) MENX-affected rats were treated with PEG vehicle (Placebo) or with two doses of BEZ235 by oral gavage for 2 weeks. Tissues were collected, fixed in performed using specific antibodies against P-S6 (Ser240/244), Ki67, NuSAP, CD31. VEGFA, active caspase-3 or Annexin V). Cell nuclei were counterstaine with DAPI. Scale bars: $50 \mu \mathrm{m}$

(B) Quantification of the IF signal with Image $J$ software for the antibodies again P-S6, CD31, VEGFA, active caspase 3 , and Annexin V. For Ki67, the percentage of the positive nuclei were counted.

B $\underset{\text { (Ser 240/244) }}{\text { pS6 }}$
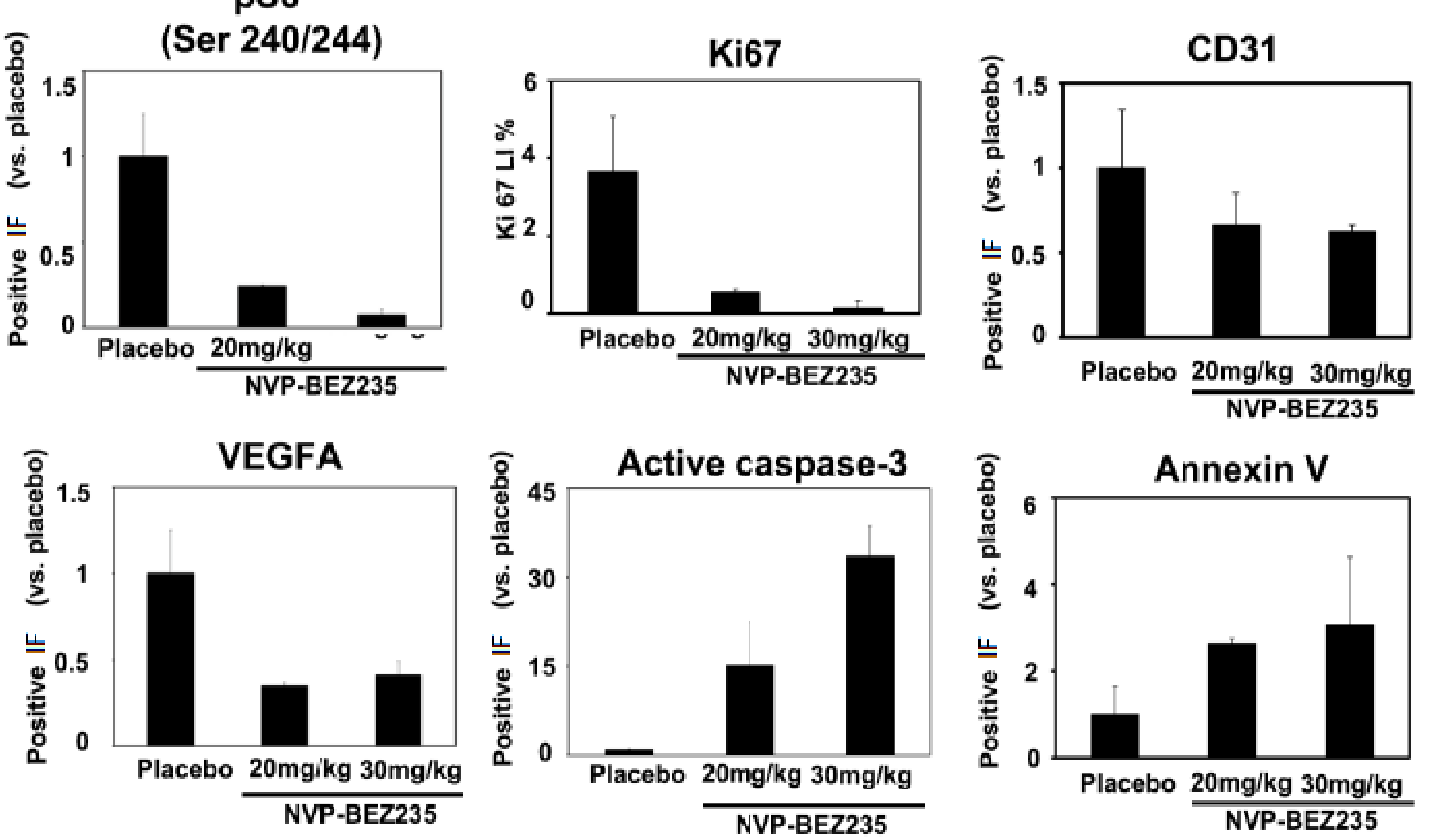

MPC cells resistant to BEZ235 do not suppress NET expression

Figure 6. The expression of NET in MPC and MPCR cells afte BEZ235 treatment. (A) Colony formation assay of MPC and MPCR cells A DMso NVP-BEZ235 5 treated with DMSO or $1 \mu \mathrm{M}$ BEZ235 for 2 weeks. Cells were then fixed and stained with crystal violet. Representative plates are shown. (B) MPC and MPCR cells were incubled with DMSO or with 1 MM BEZ235 cells were incubated with DMSO or with $1 \mu \mathrm{M}$ BEZ235 for 48h. Proteins were extracted and the expression of
NET, p-AKT, p-S6, NuSAP, Annexin $V$ and $\alpha$-tubulin NET, p-AKT, p-S6, NuSAP, Annexin $V$ and $a$-tubuln

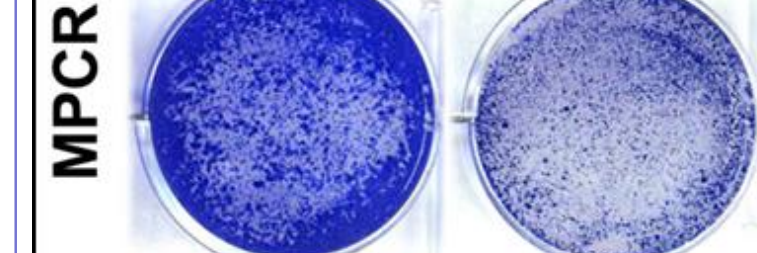
C cetected by western treated as in $B$ and

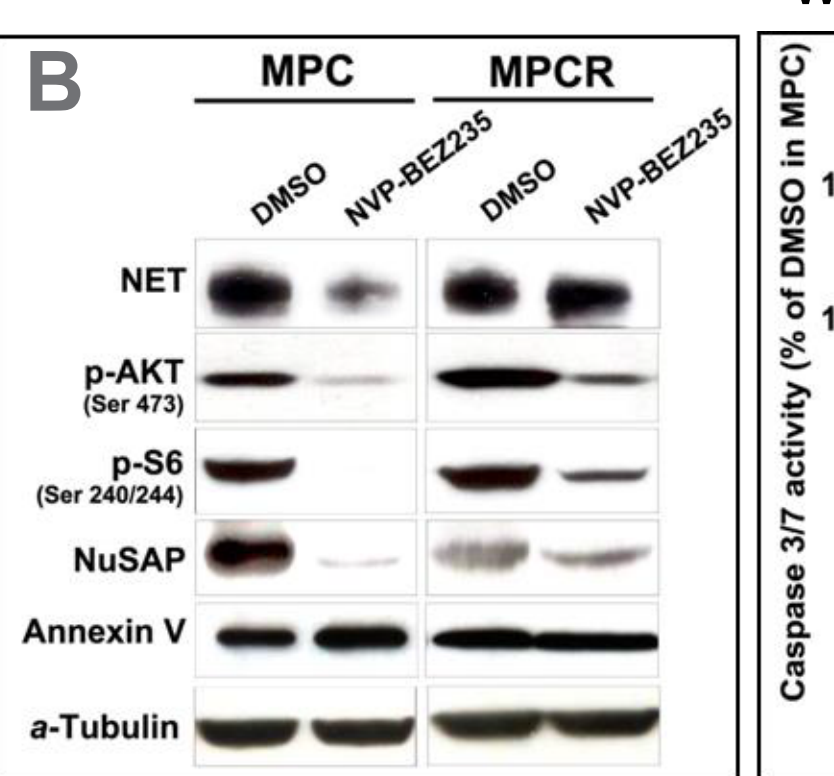
$-3 / 7$ activity. Shown is the average of the relative expression values nomalyed exprestion valus nom cells arbitrarily set to 110 (ans

treatment and the expression of NET, P-AKT and P-S6 was monitored by WB.

\section{References:}

Cantrell DA. Phosphoinosilide 3kinase signalling pathways. J Cell Sci 2001:114:1439-45.

Majumder PK, Febbo PG, Bikoff R, Berger R, Xue Q, McMahon LM, et al. mTOR inhibition

HIF-1-dependent $P$

\section{IDC Institute for Diabetes and Cancer} response of rat PCs BEZ235. Apparent diffusion coefficient (ADC) values were obtained by performing DW-MRI before (pre) and after (post) treatment of mutant rats with NVP-
BEZ235 for 14 days.

SIc6a2/NET (norepinephrine transporter) reduction after BEZ235 treatment in rat PCs and primary cells A $\quad B \quad$ Rat PC

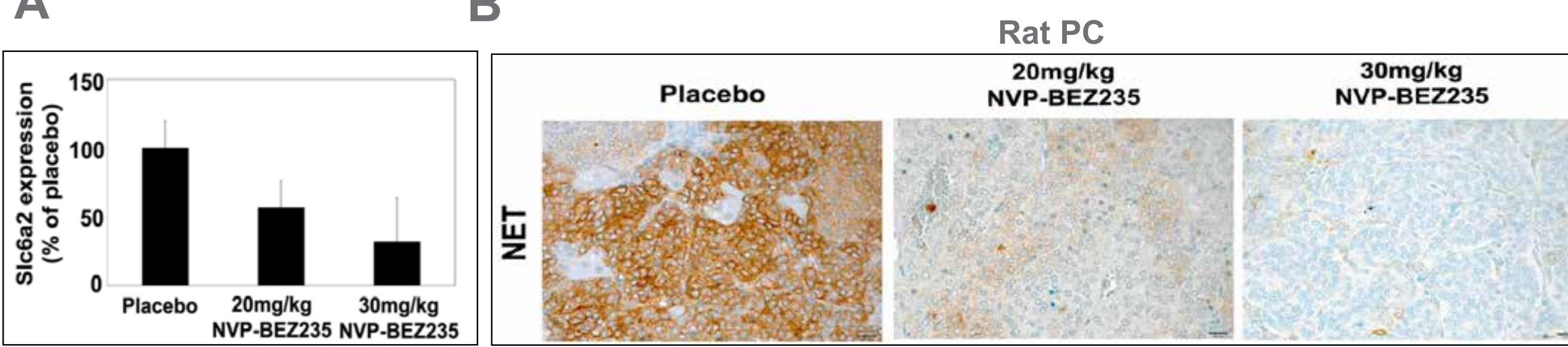

Figure 4. NVP-BEZ235 reduces SIc6a2/NET expression in rat $\mathrm{PCs}$ in vivo and in vitro. (A) GRT-PCR was performed using TaqMan primer and probe sets specific to rat S/C6a2. The obtained relati was normalized against the average expression of placebo-treated tissues arbitrarily set to 100. (B) PCs were collected after 14 days of daily placebo or BEZ235 administration and analyzed by immunostaining for NET expression. Scale bars: $20 \mu \mathrm{m}$. (C) In vitro cultures of rat primary PC cells were treated and immunofluorescent or with NVP-BEZ235 $(1 \mu \mathrm{M})$ for 3 days. Then they were fixed were counterstained with DAPI. Scale bars: $50 \mu \mathrm{m}$.

Reduced uptake of 18F-LMI11195 by PCCs after BEZ235 treatment in vivo

Figure 7.Functional imaging with 18F-LMl1195 to monitor the response of rat PCCs to BEZ235 in vivo.

PET imaging was performed before (Pre) and after (Post) treatment with placebo (PEG) or BEZ235 for 14 days. (A) Representative images of the PET scans obtained Qune adrenal glands.
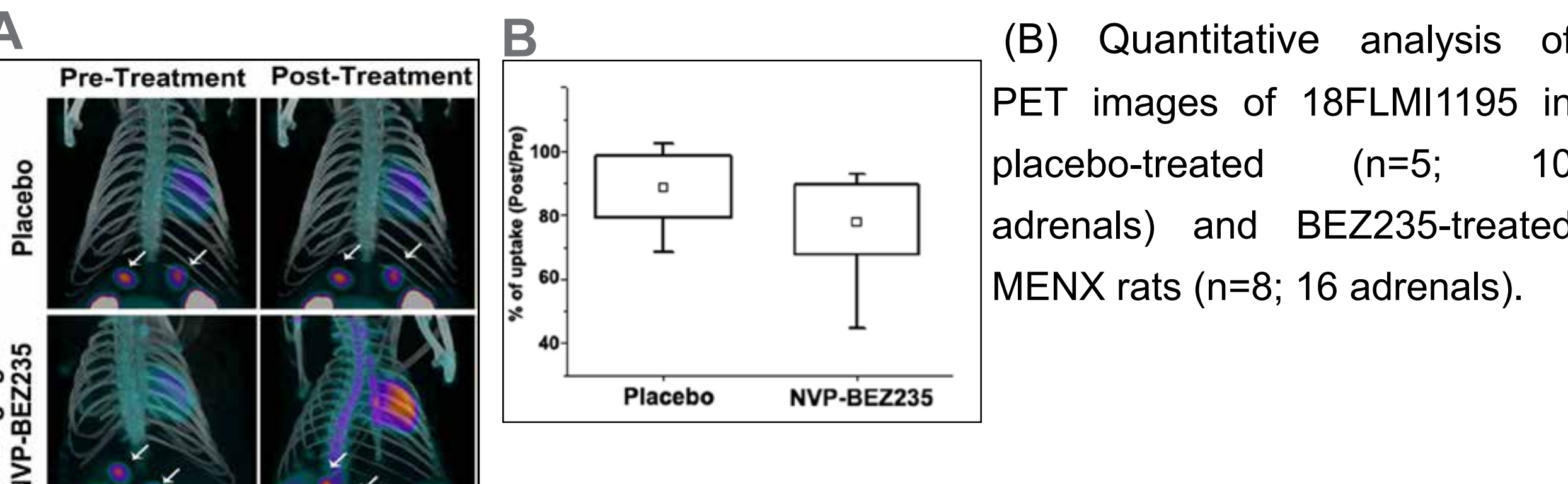

Conclusions :

Our findings establish PI3K/mTOR inhibition as an effective therapeutic option for PC. NET expression seems to be a predictive biomarker of the response of PC cells to a blockade of PI3K and mTOR signaling, which can be assessed by functional imaging 